POS PROCEEDINGS

\title{
Computing the muon anomalous magnetic moment using the hybrid method with physical quark masses
}

Matt Spraggs $^{\star a}$, Peter Boyle $^{b}$, Luigi Del Debbio ${ }^{b}$, Taku Izubuchic $^{c, d}$, Andreas Jüttner $^{a}$, Christoph Lehner ${ }^{c}$, Kim Maltman $^{e, f}$, Marina Krstić Marinkovićc ${ }^{\prime}$, Antonin Portelli ${ }^{a, b}$

${ }^{a}$ School of Physics and Astronomy, University of Southampton, Southampton SO17 1BJ, UK

${ }^{b}$ SUPA, School of Physics, The University of Edinburgh, Edinburgh EH9 3JZ, UK

${ }^{c}$ Physics Department, Brookhaven National Laboratory, Upton, NY 11973, US

${ }^{d}$ RIKEN-BNL Research Center, Brookhaven National Laboratory, Upton, NY 11973, US

${ }^{e}$ Department of Physics and Astronomy, York University, Toronto, Ontario, M3J 1P3, Canada

${ }^{f}$ CSSM, University of Adelaide, Adelaide, SA 5005, Australia

${ }^{g}$ CERN, Physics Department, 1211 Geneva 23, Switzerland

Email: m.spraggsesoton.ac.uk

\begin{abstract}
I present our work on the leading strange quark-connected contribution to the muon anomalous magnetic moment using RBC/UKQCD physical point domain wall fermion ensembles. I also present preliminary results of a computation of the light quark-connected contribution using a similar approach.
\end{abstract}

34th annual International Symposium on Lattice Field Theory

24-30 July 2016

University of Southampton, UK

\footnotetext{
* Speaker.
} 


\section{Introduction}

The anomalous magnetic moment of the muon, $a_{\mu}$, is one of the most accurately determined quantities in particle physics, with an accuracy of the order of one part per million [1]. There is currently a $3 \sigma$ to $4 \sigma$ tension between the experimental and theoretical determinations of this quantity. The new muon $g-2$ experiments at Fermilab and J-PARC aim to reduce the experimental uncertainty by a factor of four, making a reduction in the theoretical error desirable. The leadingorder (LO) hadronic contribution is the main source of this uncertainty, and is currently computed using $\sigma\left(e^{+} e^{-} \rightarrow\right.$ hadrons $)$ data [2,3]. A first-principles verification of this quantity is therefore very enticing. Here we present computations of selected connected contributions to this quantity.

Thanks in part to the work in [4] the individual contributions to $a_{\mu}^{(2) \text { had }}$ from the various possible Wick contractions may be considered separately. For a given Wick contraction $C$ one may consider the associated contribution to $a_{\mu}^{(2) \text { had }}$, denoted $a_{\mu}^{(2) \text { had, } C}$. This can be comptued as follows [5]:

$$
a_{\mu}^{(2) \text { had, } C}=\left(\frac{\alpha}{\pi}\right)^{2} \int_{0}^{\infty} \mathrm{d} Q^{2} \hat{\Pi}^{C}\left(Q^{2}\right) f\left(Q^{2}\right),
$$

where $\alpha$ is the QED coupling, $\hat{\Pi}^{C}\left(Q^{2}\right)=4 \pi^{2}\left(\Pi^{C}\left(Q^{2}\right)-\Pi^{C}(0)\right)$ is the infrared subtracted hadronic vacuum polarisation (HVP) form factor computed from an individual Wick contraction $C$, and $f$ is the integration kernel derived in perturbation theory with a singularity at $Q^{2}=0$ [5]. The integrand is highly peaked near $Q^{2} \approx m_{\mu}^{2} / 4$ such that the final value of $a_{\mu}^{(2) \text { had }}$ is highly sensitive to variations in $\hat{\Pi}\left(Q^{2}\right)$ for small $Q^{2}$.

In what follows we summarise our recent work on the strange connected contribution to $a_{\mu}^{(2) \text { had }}$, denoted $a_{\mu}^{(2) \text { had,s }}$, before turning to the light connected contribution. We finish with a brief discussion of ongoing work concerning the light and charm contributions.

\section{Simulation Details}

Simulations have been performed on the two $2+1$ flavour Möbius domain wall fermion (MDWF) ensembles with near-physical pion masses described in [6]. We compute the quark-connected vector-vector two-point function for flavour $f, C_{\mu \nu}^{f}$, using $\mathbb{Z}_{2}$ wall sources and MDWF localconserved currents, i.e.:

$$
C_{\mu v}^{f}(x)=Z_{V} Q_{f}^{2}\left\langle\mathscr{V}_{\mu}^{f}(x) V_{v}^{f}(0)\right\rangle,
$$

where $Z_{V}$ is the vector renormalisation constant, $V_{v}(x)=\bar{\psi}(x) \gamma_{\nu} \psi(x)$ is the local current and $\mathscr{V}_{\mu}(x)$ the conserved current for MDWF, as described in [6]. For details of the specific measurements used to compute $a_{\mu}^{(2) \mathrm{had}, s}$ we refer the reader to [7].

When computing $C_{\mu \nu}^{l}$ we performed measurements on the $48^{3} \times 96$ ensemble of [6] only, using diluted stochastic $\mathbb{Z}_{2}$ wall sources and subspace deflation to achieve an improvement in the signalto-noise ratio of our measurements. In addition, $C_{\mu \nu}^{l}$ is computed using local currents at both the source and the sink with unitary light quark masses. For full detail see [8].

\section{Analysis}

Our general analysis approach is described in detail in [7] and [9], so what follows will be a brief summary only. We implemented a variety of analysis strategies in order to ascertain the dependence of $a_{\mu}^{(2) \text { had,s }}$ and $a_{\mu}^{(2) \mathrm{had}, l}$ on the analysis technique. 


\subsection{HVP Computation}

We compute the HVP form factor by Fourier-transforming the vector-vector two-point function and exploiting the lattice decomposition of the HVP tensor. We remove a potential source of lattice cut-off effects by considering only the diagonal components of the HVP tensor where $\hat{Q}_{\mu}=0$ [10]. Combining this result with a lattice Fourier transform produces the following expression for the HVP form factor:

$$
\Pi\left(\hat{Q}^{2}\right)=\frac{a^{4}}{3} \sum_{t, \mathbf{x}, i}\left(\frac{\mathrm{e}^{\mathrm{i} Q_{t} t}-1}{\hat{Q}_{t}^{2}}\right) C_{i i}(\mathbf{x}, t),
$$

where $Q_{t}$ is the temporal component of the photon momentum and the subtraction of unity within the brackets effectively subtracts the zero-mode [11]. In the infinite volume limit the zero-mode vanishes, and subtracting it greatly reduces the noise in the low- $Q^{2}$ region. In the strange case we observed a factor of 48 improvement in the relative error on $\Pi\left(\hat{Q}^{2}\right)$ at the smallest non-zero value of $Q^{2}$ when $\mathbb{Z}_{2}$ wall sources were used.

When computing the contribution to $\Pi\left(\hat{Q}^{2}\right)$ from light quarks we also include a subtraction of the second time moment of $\Pi_{\mu \nu}$. This automatically subtracts $\Pi\left(Q^{2}=0\right)$ to produce directly $\hat{\Pi}$, removing unphysical quadratic divergences introduced by using a non-transverse correlation function. We combine this with a truncation of the sum in Equation (3.1) to reduce the noise introduced into the HVP at the expense of an additional systematic to be quantified. For further details, see [8].

\subsection{Hybrid Method}

We used the hybrid method as described in [12]. This method consists of partitioning the integrand in (1.1) into three non-overlapping adjacent regions using cuts at low- and high- $Q^{2}\left(Q_{\text {low }}^{2}\right.$ and $Q_{\text {high }}^{2}$ ). We then compute the integrand for the low-, mid- and high- $Q^{2}$ regions using a parametrisation of the HVP, direct numerical integration and perturbation theory, respectively. Our parametrisation of the low- $Q^{2}$ region allows us to compute $\Pi(0)$ and in turn $\hat{\Pi}\left(Q^{2}\right)$. Restricting the use of an HVP parametrisation to the low- $Q^{2}$ region allows us to minimise systematic effects [12].

We parametrise the HVP using both Padé approximants and conformal polynomials, the details of which may be found in [9].

We use two techniques for constraining the low- $Q^{2}$ models: $\chi^{2}$ minimisation and discrete time moments [13]. At our current level of precision we can only perform uncorrelated fits due to the very singular nature of the covariance matrix.

We also implement a discrete version of the continuous time moments method described in [13]. Specifically we use a general discrete derivative operator $\hat{\Delta}_{Q_{t}}$ in place of the continuous partial derivative $\partial_{Q_{t}}$, allowing us to write

$$
\left.\hat{\Delta}_{Q_{t}}^{(2 n)}\left(\frac{a^{4}}{3} \sum_{t, \mathbf{x}, i} \mathrm{e}^{-\mathrm{i} Q_{t} t} C_{i i}(\mathbf{x}, t)\right)\right|_{Q_{t}=0}=\left.\hat{\Delta}_{Q_{t}}^{(2 n)}\left(\hat{Q}_{t}^{2} \Pi\left(\hat{Q}_{t}^{2}\right)\right)\right|_{Q_{t}=0} .
$$

Inserting one of the above parametrisations of the HVP allows us to set up a system of equations that can be solved to constrain the parametrisation. Our use of discrete derivatives avoids the assumption of an infinite volume that would be required in the continuous case.

\subsection{Continuous Momenta}

We can also compute the HVP directly at any momentum by inserting this momentum di- 
rectly into the lattice Fourier transform $[14,15]$. Specifically, defining $Q_{t}=\frac{2 \pi n_{t}}{T}$, we can let $n_{t} \in[-T / 2, T / 2)$. This allows $a_{\mu}^{(2) \text { had }}$ to be computed without a parametrisation of the HVP.

This procedure incurs the possibility of introducing finite volume effects into $a_{\mu}^{(2) \text { had }}$. However, we found in [7] that these were negligible in the case of $a_{\mu}^{(2) \text { had, } s}$. Furthermore, it can be demonstrated that these effects are exponentially suppressed by the temporal extent of the lattice [14].

\section{Results}

\subsection{Strange Quark-Connected Contribution}

In computing $a_{\mu}^{(2) \text { had,s }}$ using the hybrid method we used six different parametrisations of the HVP: $P_{3}^{0.5 \mathrm{GeV}}, P_{4}^{0.5 \mathrm{GeV}}, P_{3}^{0.6 \mathrm{GeV}}, P_{4}^{0.6 \mathrm{GeV}}, R_{1,1}$ and $R_{1,2}$. We also scanned three low cuts: $0.5 \mathrm{GeV}^{2}$, $0.7 \mathrm{GeV}^{2}$ and $0.9 \mathrm{GeV}^{2}$. We use a single high cut of $5 \mathrm{GeV}^{2}$ in both the hybrid and continuous momentum methods. In this second method we used a step size of 0.005 for $n_{t}$.

As we described in [7], the use of both partially quenched and unitary strange quark masses in our measurements allows us to perform a two-dimensional linear fit in both $a^{2}$ and the relative deviation of the strange mass from its physical value. Our use of both partially quenched and unitary measurements highlighted the mass-dependence in $a_{\mu}^{(2) \text { had,s }}$ : including the former set of measurements resulted in a shift in the value of $a_{\mu}^{(2) \text { had,s }}$ from approximately $50 \times 10^{-10}$ to $53 \times 10^{-10}$.

Figure 1 demonstrates the various values of $a_{\mu}^{(2) \text { had,s }}$ computed in this analysis. Good agreement is found between all values of $a_{\mu}^{(2) \mathrm{had}, s}$. This suggests that the systematic error resulting from the various analysis techniques is small compared to the statistical uncertainty. In addition, the distribution of results is in accordance with the findings of [16], which indicated that greater systematic uncertainties should be expected where low order Padé approximants and conformal polynomials are used in combination with large $Q_{\text {low }}^{2}$.

We anticipate finite volume effects to be negligible in the case of the strange quark-connected contribution. Since our computations are performed in the limit of isospin symmetry, isospin and $G$-parity will be conserved quantities within the HVP. We therefore argue that it is not possible for the quark-connected strange HVP to couple to the two pion ground state associated with the light HVP. Instead we expect finite volume effects to be proportional to $\mathrm{e}^{-m_{K} L}$, where $m_{K} L \approx 13.8$ for the ensembles used in this study.

\subsection{Light Quark-Connected Contribution}

We adopted a similar approach in computing $a_{\mu}^{(2) \text { had,l} l}$ to that used in computing $a_{\mu}^{(2) \text { had,s }}$. We again applied the hybrid method, this time using the six parametrisations: $R_{0,1}, R_{1,1}, R_{1,2}, P_{2}^{2 m_{\pi}}$, $P_{3}^{2 m_{\pi}}$ and $P_{4}^{2 m_{\pi}}$. We used the same low cuts as in the strange case, i.e. $0.5 \mathrm{GeV}^{2}, 0.7 \mathrm{GeV}^{2}$ and $0.9 \mathrm{GeV}^{2}$. In this case we used a high cut of $7 \mathrm{GeV}^{2}$. Our application of the continuous momenta approach also used a step of 0.005 for $n_{t}$.

Figure 2 illustrates the various values of $a_{\mu}^{(2) \text { had, } l}$ computed as part of our analysis. The values computed here are derived from an HVP form factor computed by truncating the sum in Equation (3.1) at $t=25$. We anticipate that the variation of the point of truncation can be incorporated into our analysis to determine the systematic error arising from this approach.

Although the results in Figure 2 are not derived from the complete HVP, one can still observe some of the conclusions drawn by the authors of [16]. Most notable of these is the much stronger 


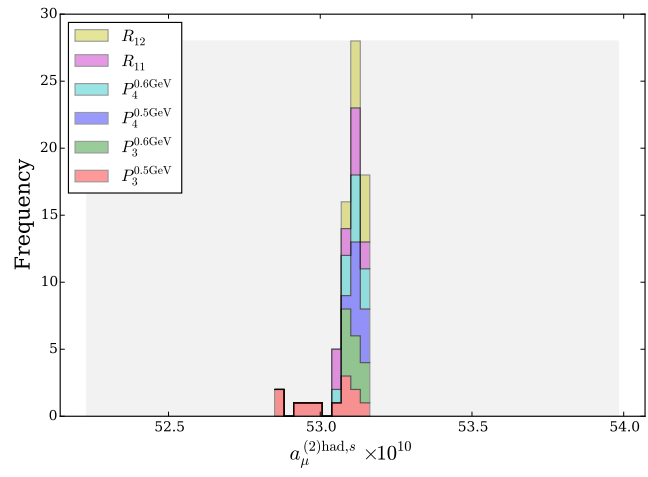

(a)

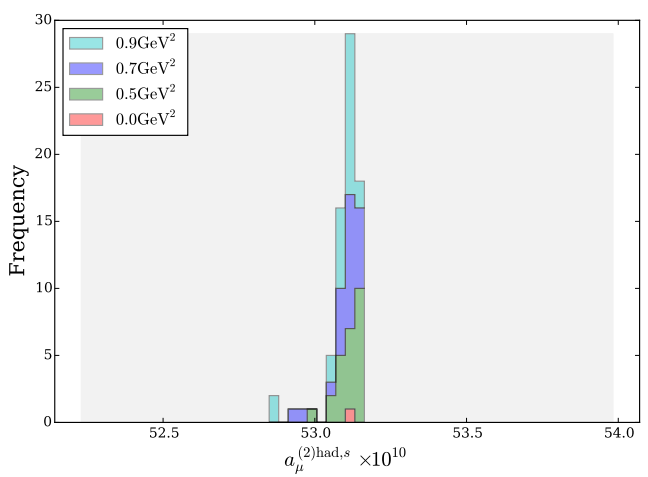

(c)

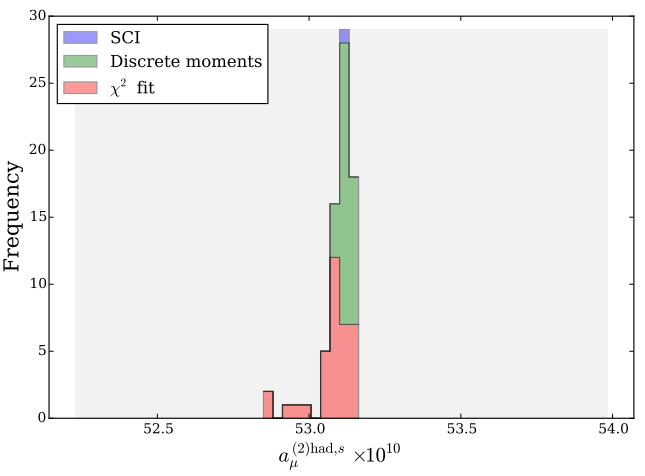

(b)

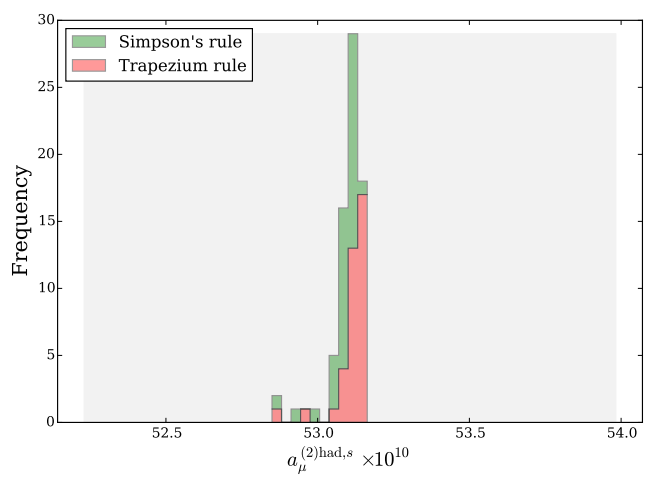

(d)

Figure 1: Stacked histograms describing the 73 values of $a_{\mu}^{(2) \text { had,s }}$ computed using the various analysis techniques, colour coded by the low- $Q^{2}$ parametrisation (panel (a)), the method used to match these parametrisations (panel (b)), $Q_{\text {low }}^{2}$ (panel (c)) and the numerical method used to integrate the mid- $Q^{2}$ region (panel (d)). The large grey band illustrates the final statistical error in the result.

dependence of $a_{\mu}^{(2) \text { had, } l}$ on the value of $Q_{\mathrm{low}}^{2}$, which is most apparent in the case of the lowestorder conformal polynomial. In addition, the much greater variation between values of $a_{\mu}^{(2) \text { had, } l}$ as compared to the analysis of $a_{\mu}^{(2) \mathrm{had}, s}$ is in accordance with the findings of [16]. The greater degree of curvature in the light HVP over the strange means that the systematic error in any particular value of $a_{\mu}^{(2) \text { had, } l}$ computed using this method will be correspondingly greater.

The preliminary nature of the results for $a_{\mu}^{(2) \text { had, } l}$ shown here cannot be understated. The cut-off effects in our result remain unquantified. Finite volume effects are currently observed to be consistent with the predictions of NLO finite volume chiral perturbation theory [8, 17], and a designated study of various lattice volumes with a fixed temporal extent is underway.

\section{Summary}

We have computed the strange contribution to the anomalous magnetic moment of the muon using domain wall fermions with physical quark masses. We have also computed a set of preliminary values of $a_{\mu}^{(2) \text { had, } l}$. We used a variety of analysis techniques, in particular the hybrid method proposed in [12] and continuous momenta [14]. These variations, along with isospin and $G$-parity 


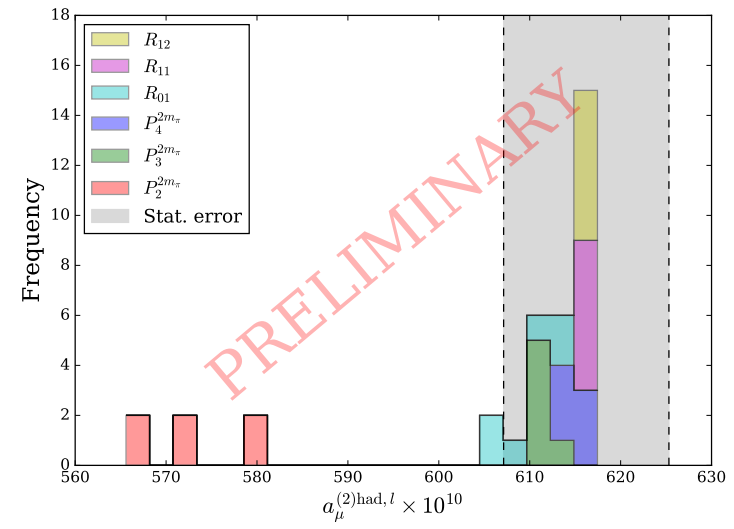

(a)

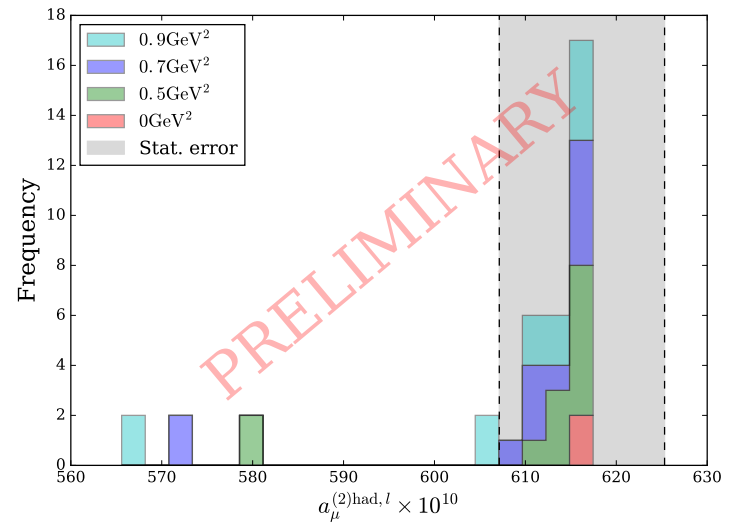

(b)

Figure 2: Stacked histograms illustrating the various values of $a_{\mu}^{(2) \text { had, } l}$ computed as part of this analysis, colour coded by the low- $Q^{2}$ parametrisation (panel (a)) and the low- $Q^{2}$ cut (panel (b)).

conservation arguments, have allowed us to compute a systematic error on our value of $a_{\mu}^{(2) \text { had, } s}$. We hence state $a_{\mu}^{(2) \text { had,s }}=53.1(9)\left(\begin{array}{l}+1 \\ -3\end{array}\right) \times 10^{-10}$ as our final value, which is in good agreement with the determination by HPQCD [13] and well within the error requirements of a $1 \%$ total error on $a_{\mu}^{(2) \text { had }}$.

Our work on the light contribution is ongoing, with the quantification of finite volume effects remaining our chief concern. Second to this we must determine the extent of the cut-off effects within our results, and it may be possible to draw on knowledge from our computation of $a_{\mu}^{(2) \text { had,s }}$.

Following on from our success in computing the quark-disconnected contribution to $a_{\mu}^{(2) \text { had }}$ earlier in 2016 [18], our focus now lies in computing the remaining contributions to $a_{\mu}^{(2) \text { had }}$ required for a total value with sub-percent precision. Specifically, we are actively investigating the charm contribution and determining the extent of corrections from isospin breaking [19].

\section{Acknowledgements}

This work is part of a programme of research by the RBC/UKQCD collaboration. This research was funded by the European Research Council under the European Community's Seventh Framework Programme (FP7/2007-2013) ERC grant agreement No 279757. P.B. acknowledges Wolfson Fellowship WM160035, an Alan Turing Fellowship and STFC grants ST/P000630/1, ST/M006530/1, ST/L000458/1, ST/L000458/1, ST/K005790/1, ST/K005804/1, ST/L000458/1. L.D.D. is supported by STFC, grant ST/L000458/1, and the Royal Society, Wolfson Research Merit Award, grant WM140078. A.J. wishes to acknowledge STFC grants ST/L000296/1 and ST/J000396/1. A.P. is supported by STFC grants ST/L000296/1 and ST/L000458/1. M.S. is funded by an EPSRC Doctoral Training Centre grant (EP/G03690X/1). The calculations reported here have been done on the DiRAC Bluegene/Q computer at the University of Edinburgh's Advanced Computing Facility.

\section{References}

[1] F. Jegerlehner and A. Nyffeler. The Muon g-2. Phys. Rept. 477 pp. 1-110 (2009) 
[2] M. Davier, A. Hoecker, B. Malaescu and Z. Zhang. Reevaluation of the Hadronic Contributions to the Muon $g-2$ and to $\alpha\left(M_{Z}\right)$. Eur. Phys. J. C71 p. 1515 (2011). [Erratum: Eur. Phys. J.C72,1874(2012)]

[3] K. Hagiwara et al. $(g-2)_{\mu}$ and $\alpha\left(M_{Z}^{2}\right)$ re-evaluated using new precise data. J. Phys. G38 p. 085003 (2011)

[4] M. Della Morte and A. Jüttner. Quark disconnected diagrams in chiral perturbation theory. JHEP 11 p. 154 (2010)

[5] T. Blum. Lattice calculation of the lowest order hadronic contribution to the muon anomalous magnetic moment. Phys. Rev. Lett. 91 p. 052001 (2003)

[6] T. Blum et al. (RBC, UKQCD). Domain wall QCD with physical quark masses. Phys. Rev. D93(7) p. 074505 (2016)

[7] T. Blum et al. (RBC/UKQCD). Lattice calculation of the leading strange quark-connected contribution to the muon $g-2$. JHEP 04 p. 063 (2016)

[8] C. Lehner. Disconnected and light connected hvp contributions to the muon anomalous magnetic moment (2016). Presented at The 34th International Symposium on Lattice Field Theory

[9] M. Spraggs et al. The strange contribution to $a_{\mu}$ with physical quark masses using Möbius domain wall fermions. PoS LATTICE2015 p. 106 (2016)

[10] P. Boyle, L. Del Debbio, E. Kerrane and J. Zanotti. Lattice Determination of the Hadronic Contribution to the Muon $g-2$ using Dynamical Domain Wall Fermions. Phys. Rev. D85 p. 074504 (2012)

[11] D. Bernecker and H. B. Meyer. Vector Correlators in Lattice QCD: Methods and applications. Eur. Phys. J. A47 p. 148 (2011)

[12] M. Golterman, K. Maltman and S. Peris. New strategy for the lattice evaluation of the leading order hadronic contribution to $(g-2)_{\mu}$. Phys.Rev. D90(7) p. 074508 (2014)

[13] B. Chakraborty et al. (HPQCD). Strange and charm quark contributions to the anomalous magnetic moment of the muon. Phys. Rev. D89(11) p. 114501 (2014)

[14] L. Del Debbio and A. Portelli (to appear)

[15] A. Portelli and L. Del Debbio. Finite volume hadronic vacuum polarisation at arbitrary momenta (2015). Presented at The 33rd International Symposium on Lattice Field Theory

[16] M. Golterman, K. Maltman and S. Peris. A Hybrid Strategy for the Lattice Evaluation of the Leading Order Hadronic Contribution to $(g-2)_{\mu}$. Nucl. Part. Phys. Proc. 273-275 pp. 1650-1656 (2016)

[17] C. Aubin et al. Finite-volume effects in the muon anomalous magnetic moment on the lattice. Phys. Rev. D93(5) p. 054508 (2016)

[18] T. Blum et al. (RBC-UKQCD). Calculation of the hadronic vacuum polarization disconnected contribution to the muon anomalous magnetic moment. Phys. Rev. Lett. 116(23) p. 232002 (2016)

[19] V. Gülpers and J. Harrison (To appear). This proceedings. 